\title{
Charmonium spectral functions in quark-gluon plasma from lattice QCD with large spatial volume
}

\author{
Chiho Nonaka* \\ Kobayashi-Maskawa Institute for the Origin of Particles and the Universe (KMI), \\ Department of physics, Nagoya University, Furo-cho, Chikusa-ku, Nagoya 464-8602, Japan \\ E-mail: nonaka@hken.phys.nagoya-u.ac.jp
}

\section{Masayuki Asakawa}

Department of Physics, Osaka University, Toyonaka 560-0043, Japan

E-mail: yuki@kern.phys.sci.osaka-u.ac.jp

\section{Takeyuki Hoshino}

Department of Physics, Nagoya University, Furo-cho, Chikusa-ku, Nagoya 464-8602, Japan

\section{Masakiyo Kitazawa}

Department of Physics, Osaka University, Toyonaka 560-0043, Japan

E-mail: kitazawa@kern.phys.sci.osaka-u.ac.jp

\section{Yasuhiro Kohno}

Department of Physics, Osaka University, Toyonaka 560-0043, Japan

E-mail: kouno@kern.phys.sci.osaka-u.ac.jp

\begin{abstract}
We study the charmonium spectral functions in quark-gluon plasma on $64^{3} \times N_{\tau}$ quenched anisotropic lattices with anisotropy $a_{\sigma} / a_{\tau}=4$, analyzing correlation functions of charmonia by the maximum entropy method (MEM). We focus on finite momentum effects on the charmonium spectral functions in order to understand $J / \psi$ suppression mechanism in relativistic heavy ion collisions from the first principles of QCD.
\end{abstract}

The XXVIII International Symposium on Lattice Field Theory, Lattice2010

June 14-19, 2010

Villasimius, Italy

\footnotetext{
* Speaker.
} 


\section{Introduction}

Since the Relativistic Heavy Ion Collider (RHIC) at Brookhaven National Laboratory started operations in 2000, a lot of experimental data with outstanding quality have been reported. The highlights of them were (i) strong elliptic flow which suggests collectivity and thermalization are achieved; (ii) strong jet quenching which confirms that hot and dense matter is created after collisions; (iii) the quark number scaling of elliptic flow which indicates that the hot quark soup is produced [1]. Together with phenomenological studies such as relativistic hydrodynamic models, recombination models and jet energy loss mechanism these experimental results led us to the discovery of strongly correlated quark gluon plasma (QGP) at RHIC [2].

Difficulties in QGP physics exist in the fact that there are no physical observables as direct QGP indicators. Tremendous efforts have been devoted to find a clear QGP signal and to acquire the comprehensive understanding of experimental data from various physical observables. $J / \psi$ suppression has been considered as one of promising signatures for QGP production since it was proposed in 1986 [3, 4]. However recent lattice QCD calculations show that charmonia survive even above critical temperature [5, 6, 7, 8 , which presents the possibility that the scenario of $J / \psi$ suppression in relativistic heavy ion collisions may change. To understand the relativistic heavy ion collision physics we need to take into account dynamics of the fireball which is produced after collisions. Therefore the detailed study on spectral functions of charmonia with finite momentum would shed light on the understanding of the $J / \psi$ suppression mechanism in relativistic heavy ion collisions. A comprehensive study of momentum dependence of charmonium spectral functions with the first principle calculation has just started.

\section{Maximum Entropy Method}

First we prepare local interpolating operators for mesons, $O_{\Gamma}(\vec{x}, \tau)=\bar{\psi}(\vec{x}, \tau) \Gamma \psi(\vec{x}, \tau)$, where $\Gamma=\gamma_{i}(i=1,2,3)\left(=\gamma_{5}\right)$ for $J / \psi\left(\eta_{c}\right)$. Then the correlation function of the meson on a lattice is wrriten with $C(\tau, \vec{p})=\sum_{x} \exp (i \vec{p} \cdot \vec{x})\left\langle O_{\Gamma}(\vec{x}, \tau) \Gamma O_{\Gamma}^{\dagger}(\overrightarrow{0}, 0)\right\rangle$. The spectral decomposition of the correlator is given with,

$$
C(\tau, \vec{p})=\int_{0}^{\infty} d \omega A(\omega, \vec{p}) K(\tau, \omega)
$$

where $\omega$ is a real frequency and $A(\omega, \vec{p})$ and $K(\tau, \omega)$ are the spectral function and the integral kernel, $K(\tau, \omega)=\left(e^{-\tau \omega}+e^{-(1 / T-\tau) \omega}\right) /\left(1-e^{-\omega / T}\right)$, respectively. $C(\tau, \vec{p})$ in Eq. (2.1) is obtained with Monte Carlo Simulations on a lattice. This value is discretized on the temporal direction of lattice $(\sim O(10))$ and contains noise. On the other hand, the spectral function $A(\omega, \vec{p})$ is continuous. To solve this ill-posed problem and extract the spectral functions from lattice data, for the first time, the Maximum Entropy Method (MEM) was applied in Ref. [9].

The MEM is a very useful and powerful tool to analyze spectral functions from lattice QCD simulations. Because it is a statistical analysis of data with noise based on Bayes' theorem, an examination of statistical significance of the solution is indispensable. The starting point of the MEM is written with the conditional probability,

$$
P[A \mid C H]=\frac{P[C \mid A H] P[A \mid H]}{P[C \mid H]},
$$


where $A, C$ and $H$ are the spectral function, lattice data and all definitions, and prior knowledge, respectively. In Eq. [2.2), $P[A \mid C H](\propto \exp (-L))$ is the standard likelihood function, $P[A \mid H](\propto$ $\exp (\alpha S))$ is the prior probability, and $P[C \mid H]$ is a normalization constant. The prior probability is written with Shannon-Jaynes entropy $S, S=\int\left[A(\omega)-m(\omega)-A(\omega) \log \left(\frac{A(\omega)}{m(\omega)}\right)\right]$, where $m(\omega)$ is a default model, and auxiliary parameters $\alpha$. In this study the default model is used with an estimate from the perturbative QCD calculation of $A(\omega \gg 1 \mathrm{GeV})[9$. There is the possibility that it may be chosen so that the MEM errors become minimum [10]. From combination of the likelihood function and the prior probability, we obtain $P[A \mid C H] \propto \exp (\alpha S-L)$, where $\alpha$ determines the relative weight between $L$ and $S$. The most probable image of $A(\omega)$ for a given $\alpha$ is found with minimizing $P[A \mid C H](=P[\alpha \mid C m])$. The final output image of $A(\omega)$ is defined by a weighted average over $A$ and $\alpha, A(\omega)=\int d \alpha A_{\alpha}(\omega) P[\alpha \mid C m] d \alpha$. The last important step of MEM is the error analysis which is done with calculation for $A(\omega)$ averaged over some interval in $\omega$.

\section{Lattice Simulations}

The parameters of Monte Carlo simulations are the same as those in the previous study 【5] except the spatial lattice size. Our main purpose is to obtain the spectral functions of charmonia with finite momentum in RHIC energy scale where non-perturbative QCD aspects play an important role. To reach the soft scale, $1 / L_{\sigma} \sim T$, we perform Monte Carlo simulations on a larger spatial lattice. The standard plaquette gauge action and Wilson quark fermion action with quenched approximation and $\beta=7.0$ on a $64^{3} \times N_{\tau}$ anisotropic lattice are employed. The anisotropic lattice with the renormalized anisotropy $\left(\xi=a_{\sigma} / a_{\tau}=4\right)$ and the fine temporal lattice size $\left(a_{\tau}=9.75 \times 10^{-3}\right)$ realize that the minimum value of spatial momentum is $\sim 0.5 \mathrm{GeV}$ with periodic boundary condition. The fermion anisotropy is set to $\gamma_{F} \equiv \kappa_{\tau} / \kappa_{\sigma}=3.476$, where $\kappa_{\sigma}(=0.08285)$ and $\kappa_{\tau}$ are the spatial and temporal hopping parameters. Gauge configurations are generated by the pseudo heatbath and overrelaxation algorithms with a ratio 1:4. Initially, the gauge field is thermalized with 10000 sweeps, and then each configuration is separated by 1000 sweeps. Here we show our numerical results at $T=1.62 T_{c}\left(N_{\tau}=46\right)$ on 398 configurations and $T=1.87 T_{c}\left(N_{\tau}=40\right)$ on 393 configurations.

Figure 1 shows spectral functions of $\eta_{c}$ with vanishing momentum together with error bars at $T=1.63 T_{c}$ (left) and $T=1.87 T_{c}$ (right). At $T=1.62 T_{c}$ there are three peaks in the spectral function; a sharp peak at $3 \sim 4(\mathrm{GeV})$ and two broad peaks around $7 \sim 9(\mathrm{GeV})$ and $14 \sim 19$ $(\mathrm{GeV})$ which are known as unphysical states of fermion doublers [11]. From comparison with the error bars the first peak has statistical significance which indicates the existence of strong $c-\bar{c}$ correlation even above the critical temperature. On the other hand, at $T=1.87 T_{c}$ no clear peaks are found in the spectral function, which suggests that the $c-\bar{c}$ correlation disappears and $\eta_{c}$ melts between $T=1.62 T_{c}$ and $T=1.87 T_{c}$. This temperature dependence of the spectral function of $\eta_{c}$ is consistent with the previous calculation [5].

$J / \psi$ spectral functions with $p=0$ at $T=1.62 T_{c}$ (left) and $T=1.87 T_{c}$ (right) are shown in Fig. 2 At $T=1.62 T_{c}$ almost the same structure is found in the spectral function of $\eta_{c}$; one sharp peak (3 $\sim 4 \mathrm{GeV})$ and two broad peaks. From comparison with error bars the first peak has statistical significance, which indicates that $J / \psi$ survives at $T=1.62 T_{c}$. At $T=1.87 T_{c}$ the spectral function 

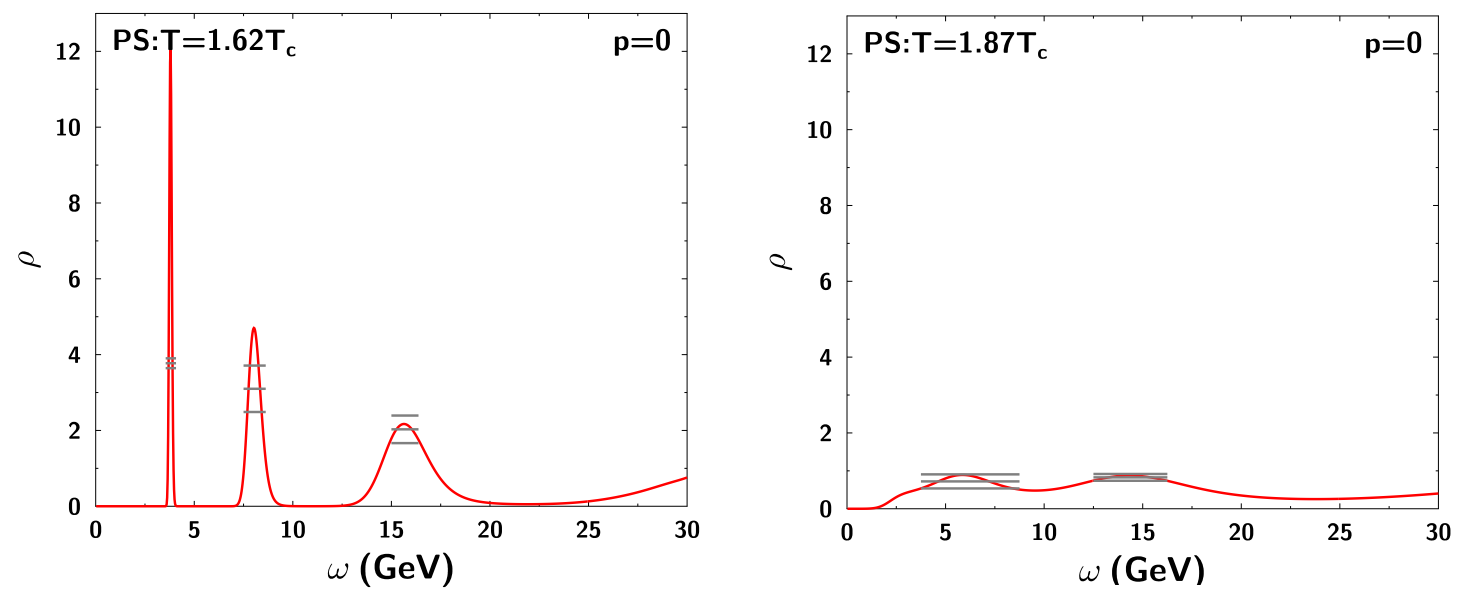

Figure 1: Spectral functions of $\eta_{c}$ with vanishing momentum at $T=1.63 T_{c}$ (left) and $T=1.87 T_{c}$ (right).

shows different structure from that at $T=1.62 T_{c},{ }^{1}$ which suggests that $J / \psi$ also survives even above the critical temperature and melts between $T=1.62 T_{c}$ and $T=1.87 T_{c}$.
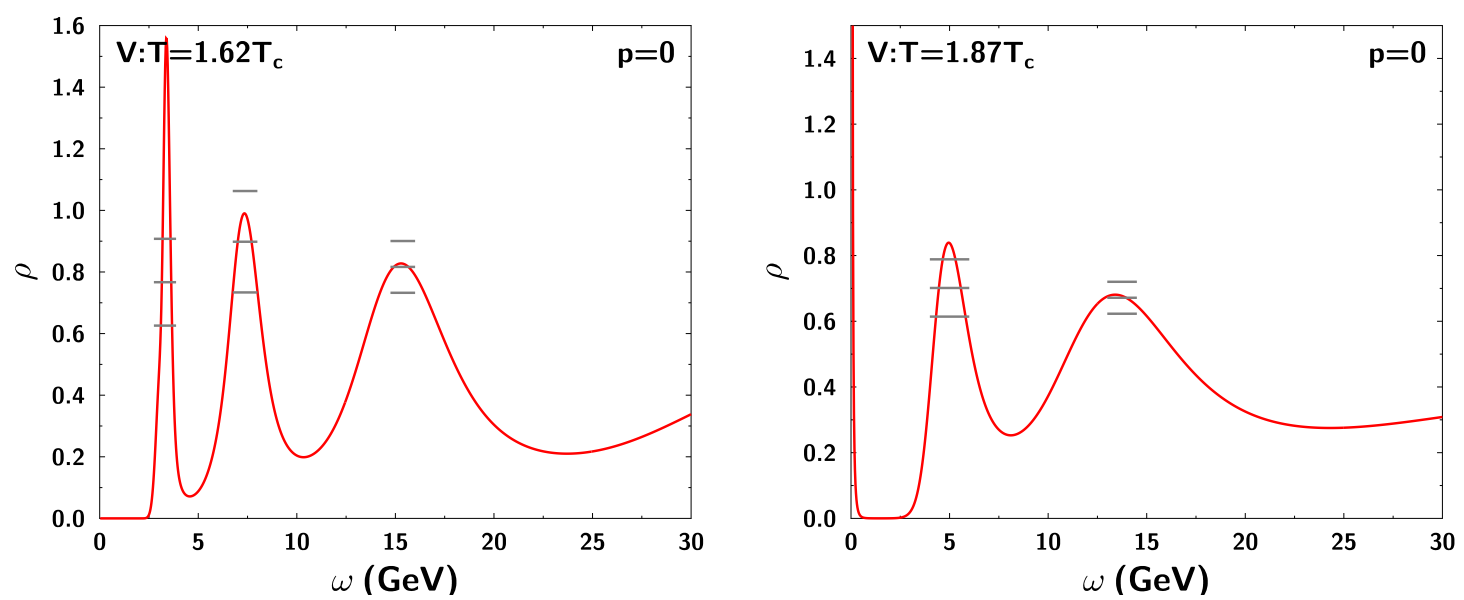

Figure 2: Spectral functions of $J / \psi$ with vanishing momentum at $T=1.63 T_{c}$ (left) and $T=1.87 T_{c}$ (right).

Next we explore the momentum dependence of spectral functions of $\eta_{c}$ and $J / \psi$ (the transverse mode) with finite momentum at $T=1.62 T_{c}$ (Figs. 3,4,5and6). Here the unit of momentum is about $0.5 \mathrm{GeV}$. For example, the actual momentum of $p=3$ is $\sim 3 \times 0.5=1.5 \mathrm{GeV}$. In both cases of $\eta_{c}$ and $J / \psi$, the three peaks are observed in their spectral functions and qualitatively the structure of them is the same as that with vanishing momentum (Figs. 3] and 57. We do not observe a significant difference between the shape of the spectral functions with finite momentum and that with zero momentum as it is found between that at $T=1.62 T_{c}$ and that at $T=1.87 T_{c}$ with vanishing momentum. Because only the first peak of the spectral functions with finite momentum has

\footnotetext{
${ }^{1}$ The spectral functions of $J / \psi$ at $T=1.87 T_{c}$ shows a rapid increase at $\omega \rightarrow 0$ which, however has no statistical siginificance. The behavior is often found in MEM analyses. To avoid a possible unstable feature at small energies a modification of the MEM algorithm is proposed in Ref. [12].
} 
statistical significance from comparison with error bars, we focus on the momentum dependence of the first peak of the spectral functions in Figs. 3 and 5 . Figs. 4 and 6 indicate that $\eta_{c}$ and $J / \psi$ at $T=1.62 T_{c}$ are stable even at higher momentum. However quantitative discrepancies in behavior of momentum dependence of spectral functions of the first peak between $\eta_{c}$ and $J / \psi$ are found. In the case of $\eta_{c}$, the strength of the first peak becomes smaller at higher momentum. The location of the first peak of spectral functions shifts to larger $\omega$ at higher momentum. On the other hand, in $J / \psi$ spectral functions, we do not find the systematic behavior in momentum dependence of the strength of the first peak. Furthermore the location of the first peak shows stronger momentum dependence compared to $\eta_{c}$.

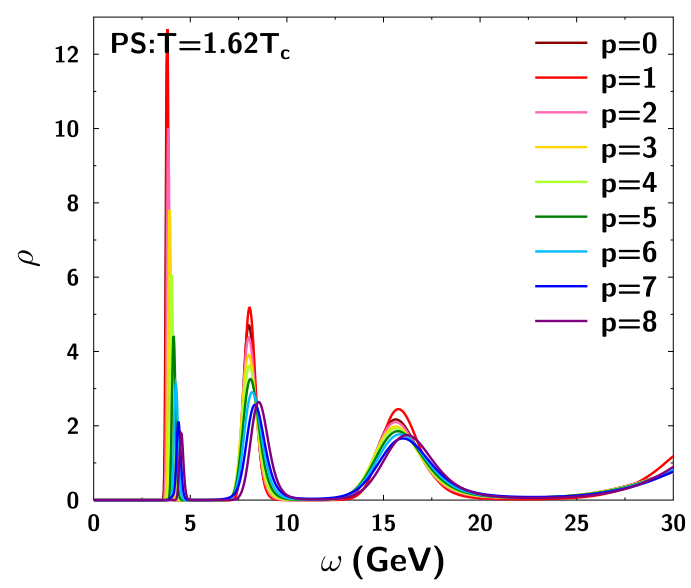

Figure 3: Momentum dependence of spectral functions of $\eta_{c}$ at $T=1.62 T_{c}$.

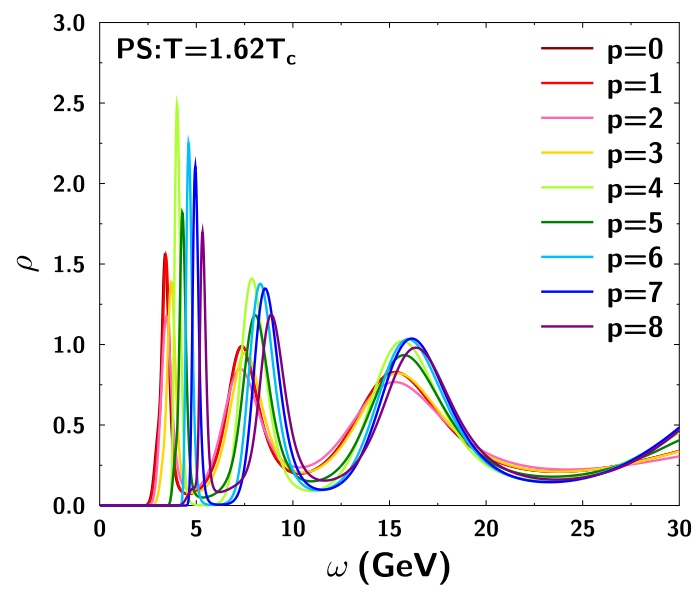

Figure 5: Momentum dependence of spectral functions of $J / \psi$ (the transverse mode) at $T=1.62 T_{c}$.

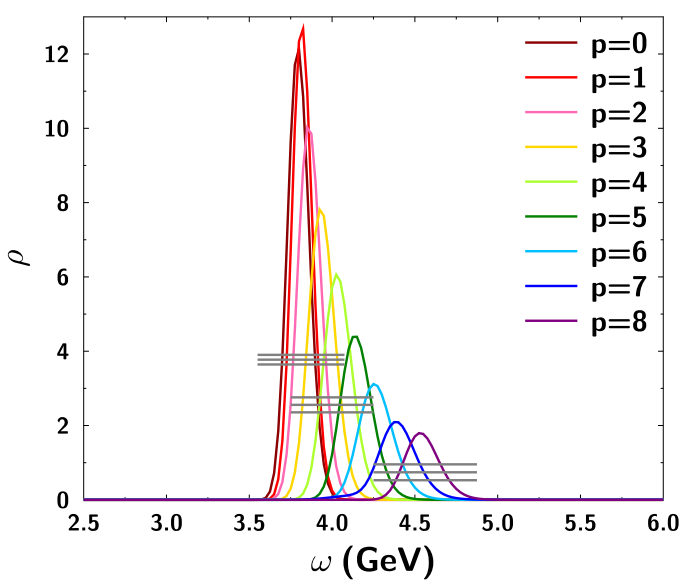

Figure 4: Momentum dependence of spectral functions of $\eta_{c}$ at $T=1.62 T_{c}$ (the first peak in Fig. 3).

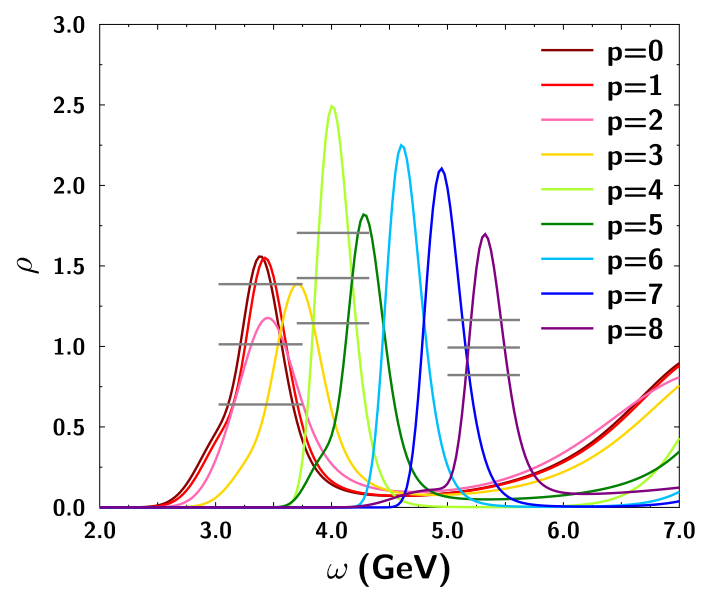

Figure 6: Momentum dependence of spectral functions of $J / \psi$ (the transverse mode) at $T=1.62 T_{c}$ (the first peak in Fig. 5).

Finally we investigate the dispersion relation of $\eta_{c}$ and $J / \psi$ (the transverse mode) at $T=$ 
$1.62 T_{c}$ to explore the medium effect in the spectral functions. Figs. 7 and 8 show the first peak of the spectral functions with $p=6,7$, and 8 . The solid circles are obtained with the dispersion relation in vacuum, $\left.\omega\right|_{v} ^{2}=\left.m^{2}\right|_{p=0}+\hat{p}_{\text {lattice }}^{2}$, where $\hat{p}_{\text {lattice }}$ is the momentum for free bosons on the lattice, $\hat{p}_{\text {lattice }}=\frac{2}{a_{\sigma}} \sin \left(p a_{\sigma} / 2\right)$ and $\left.m\right|_{p=0}$ is determined with the spectral functions of vanishing momentum at $T=1.62 T_{c}$. In the case of $\eta_{c}$, the deviation from the dispersion relation at vacuum starts to appear at $p \sim 1.5 \mathrm{GeV}$ and difference between the location of the first peak and $\omega_{v}$ increases with momentum. This indicates that the dispersion relation of $\eta_{c}$ in medium is modified from that in vacuum. On the other hand, in the transverse mode of $J / \psi$ the location of the first peak is almost the same as $\omega_{v}$ within the error bars, which implies that the dispersion relation of $J / \psi$ is not affected with medium. In order to confirm this interesting feature of charmonium spectral functions, we are investigating the longitudinal mode of vector channel and carrying out the Monte Carlo simulations at different temperatures.
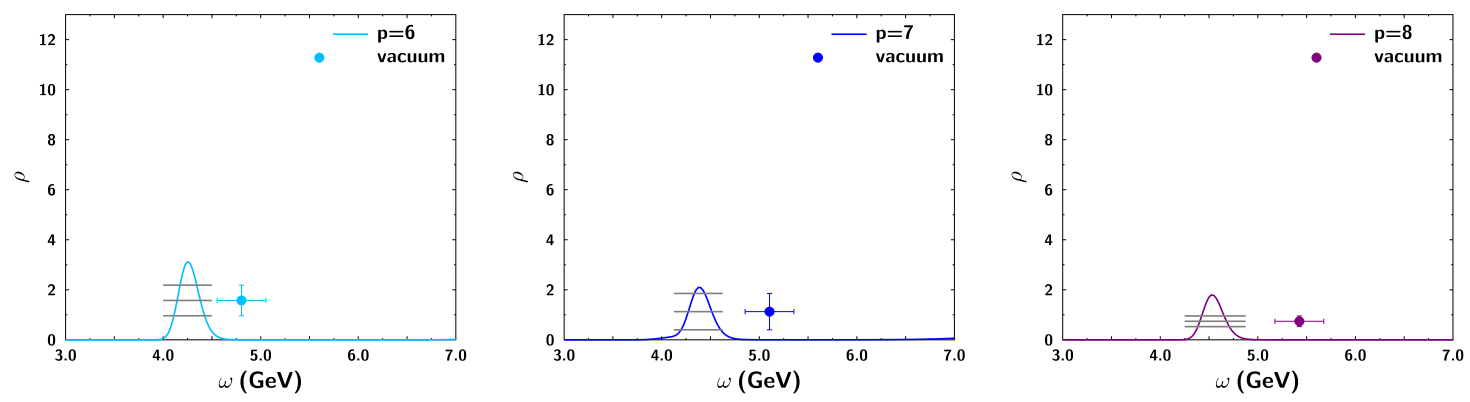

Figure 7: Spectral functions of $\eta_{c}$ at $T=1.62 T_{c}$ together with $\omega_{v}$.
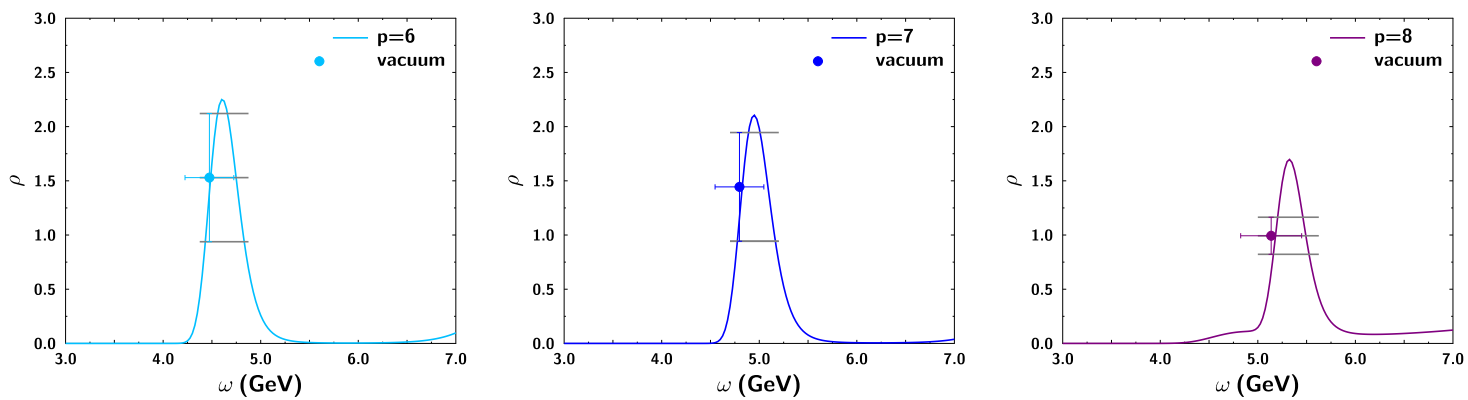

Figure 8: Spectral functions of $J / \psi$ (the transverse mode) at $T=1.62 T_{c}$ together with $\omega_{v}$.

In summary to get insight of charmonia for a QGP signal in relativistic heavy ion collisions we studied the charmonium spectral functions in quark-gluon plasma on $64^{3} \times N_{\tau}$ quenched anisotropic lattices, analyzing correlation functions of charmonia with the maximum entropy method (MEM). We found that $\eta_{c}$ and $J / \psi$ are stable above the critical temperature $T=1.62 T_{c}$ even with the finite momentum. In the case of $\eta_{c}$, the modification of dispersion relation from that of vacuum was observed. To obtain more detailed structure of charmonium spectral functions, we are carrying out calculations at other temperatures $T=0.78,1.38,1.70,1.78$ and $2.33 T_{c}$.

\section{Acknowledgement}

Lattice calculations have been performed with the PACS-CS computer in Tsukuba and Blue 
gene in KEK. This work is supported in part by JSPS Institutional Program for Young Researcher Overseas Visits, Grant-in-Aid for Young Scientists (B) (22740156) and Grant-in-Aid for Scientific Research (S) (22224003) (C.N.).

\section{References}

[1] First Three Years of Operation of RHIC, Nucl. Phys. A 757(2005)1.

[2] Quark-Gluon Plasma. New Discoveries at RHIC: Case for the Strongly Interacting Quark-Gluon Plasma. Nucl. Phys. A 750(2005)1.

[3] T. Matsui and H. Satz, Phys. Lett. B 178 (1986) 416.

[4] T. Hashimoto, K. Hirose, T. Kanki and O. Miyamura, Phys. Rev. Lett. 57 (1986) 2123.

[5] M. Asakawa and T. Hatsuda, Phys. Rev. Lett. 92 (2004) 012001 [arXiv:hep-lat/0308034].

[6] S. Datta, F. Karsch, P. Petreczky and I. Wetzorke, Phys. Rev. D 69 (2004) 094507 [arXiv:hep-lat/0312037].

[7] H. Iida, T. Doi, N. Ishii, H. Suganuma and K. Tsumura, Phys. Rev. D 74 (2006) 074502 [arXiv:hep-lat/0602008].

[8] T. Umeda, Phys. Rev. D 75 (2007) 094502 [arXiv:hep-lat/0701005].

[9] M. Asakawa, T. Hatsuda and Y. Nakahara, Prog. Part. Nucl. Phys. 46 (2001) 459 [arXiv:hep-lat/0011040].

[10] H-T. DING, these proceedings.

[11] T. Yamazaki et al. [CP-PACS Collaboration], Phys. Rev. D 65 (2002) 014501 [arXiv:hep-lat/0105030].

[12] G. Aarts, C. Allton, J. Foley, S. Hands and S. Kim, Phys. Rev. Lett. 99 (2007) 022002 [arXiv:hep-lat/0703008]. 